\title{
INVESTIGATION OF TRAFFIC FLOW DYNAMIC PROCESSES USING DISCRETE MODEL
}

\author{
Marijonas Bogdevicius, Raimundas Junevicius \\ Vilnius Gediminas Technical University, Department of Transport and Technological Equipment \\ Plytinés 27, LT-10105, Vilnius, Lithuania \\ tel.: +37052744782 \\ e-mail:marijonas.bogdevicius@vgtu.lt,raimundas@junevicius.com
}

\begin{abstract}
Modelling the process of traffic flow was previously studied from different points of view and different mathematical methods where used to describe the same process. All authors have an agreement on basic traffic flow parameters like, traffic flow density, traffic flow rate or the average speed of traffic flow. Besides, a lot of different investigations into the use of traffic flow models to deal with various problems of engineering are carried out. A comparison of different continuum models has drawn that a number of scientific works were based on fluid dynamic theory and gas - kinetic traffic flow theory. The kinetic traffic flow theory is used in 'microscopic' or "macroscopic", traffic flow models.

The kinetic traffic flow theory is used in Flötteröd G., Nagel K., Ging A., Li L., Li-qun X., Prigogine I., Herman R. works where various approaches to the similar method are discussed. The 'macroscopic' theory of traffic flows also can be developed as the hydrodynamic theory of fluids that was first introduced by Lighthill-Whitham and Richards's model. Plenty of traffic flow models are based on car-following theories supported by the analogues to Newton's equation for each individual vehicle interacting in a system of vehicles on the road. Different forms of the equation of motion give different versions of car-following models. This work presents research of traffic flow dynamic processes, as nonlinear dynamic system, by using a discrete model of traffic flow (DMTF). The main variables in DMTF are traffic flow density and speed. DMTF can be used to describe various traffic flow situations in the roads. The mathematical simulation of traffic flow is made when constant value of traffic flow speed and traffic flow rate is entered. Numerical results of traffic flow dynamics are obtained.
\end{abstract}

Keywords: traffic flow, method, dynamics, numerical results

\section{Introduction}

Modelling of such processes like traffic flow was studied previously in different points of view. These views give us different varies of mathematical approach of the same process. It also gives us difficulties to find the right method to derive physical appearance. Different authors have different views to the same phenomena and take different aspects of the same problem in the first place.

All authors have an agreement of basic traffic flow parameters, like traffic flow average speed, density or rate.

Comparing different models, it was noticed that a lot of works were based on fluid dynamic continuous and kinetic traffic flow discrete theories. Kinetic traffic flow theory is used for so called "microscopic", "macroscopic" or continues traffic flow models. Kinetic traffic flow theory is used on [5, 8-10] works. Equations of these models take different values to derive the same process. First time Kinetic theory was used by Prigogine [9], and his co-workers. They suggested an equation analogue to Boltzmann equation. This theory was criticized later by many authors like Pavery-Fontana or C. Tampier [11]. Pavery-Fontna noticed that Prigogine's model has inaccuracies comparing modelling results and physical experiments. He suggested model with vehicle-desired velocity towards which its actual velocity tends.

"Macroscopic" theory of traffic flows can also be developed as the hydrodynamic theory of fluids. First time hydrodynamic theory was presented by Lighthill-Whitham and Richards model 
$[1,3,4,6,7]$. They presented a one-dimensional model in analogue to fluid stream model. This theory was criticized by authors like Payne [11] and Daganzo, Nagatani. Nagatani proposed lattice method. Nagatani and Nakanishi model was taking into account that all vehicles move at the same time-independent speed and the same gap between vehicles. This method was improved later by taking next-nearest neighbour interaction into account [4].

A lot of traffic flow models are based on car-following theories. These theories are based on analogues to Newton's equation for each individual vehicle interacting in a system of vehicles on a road. Different forms of equation of motion give different versions of car-following models. Stimulus, from which the response may occur, may be composed of the speed of vehicle, the difference in the speeds of leading and going after vehicle, the distance-headway, etc.

From the car following, theories mostly known are follow-the-leader and optimal-velocity theories. These theories are used in literature [11, 13]. Using these methods kinetic and fluid dynamic models could be extended in critical points when the kinetic and fluid theory gives us inaccuracies comparing with experimental data.

\section{Mathematical model of rail and rail wheel contact}

The aim of this investigation is to obtain dynamic characteristics of traffic flow by using a discrete model of traffic flow (DMTF) [14] R. Junevičius, M. Bogdevičius. A discrete model of traffic flow was created in order to analyse the main traffic flow conditions in the city network.

The discrete model of traffic flow uses an equation system that takes in to account two parameters: traffic flow density and traffic flow speed. These parameters are calculated on each road point and the information from the previous and the next point of some road mesh is taken into account (Fig. 1.).

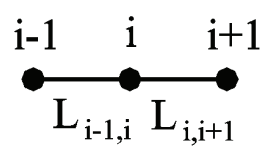

Fig. 1. Scheme to derive traffic flow values at each traffic line point

At each point " $i$ " equations 1 and 2 are derived. Equation one derives the variation of traffic flow speed and equation two derives variation of the concentration at each point $i$ :

$$
\begin{aligned}
& \dot{k}_{i}=\sum_{j=1}^{N i n} p_{i n, i, j}(t) \cdot r_{k_{i}, \text { in. i, }, j} \cdot \Phi_{k, \text { in }, i, j}\left(k_{j}\left(t-\tau_{i, j}\right), v_{j}\left(t-\tau_{i, j}\right), k_{i}(t), v_{i}(t)\right) \cdot k_{i}(t)- \\
& \sum_{j=1}^{\text {Nout }} p_{\text {out }, i, j}(t) \cdot r_{k_{i}, \text { out }, i, j} \cdot \Phi_{k, \text { out }, i, j}\left(k_{i}(t), v_{i}(t), k_{j}(t), v_{j}(t)\right) \cdot k_{i}(t), \\
& \dot{v}_{i}=\sum_{j=1}^{N \text { in }} p_{i n, i, j}(t) \cdot r_{v_{i}, \text { in. i, }, j} \cdot \Phi_{v, \text { in }, i, j}\left(k_{j}\left(t-\tau_{i, j}\right), v_{j}\left(t-\tau_{i, j}\right), k_{i}(t), v_{i}(t)\right) \cdot v_{i}(t)- \\
& \sum_{j=1}^{\text {Nout }} p_{\text {out }, i, j}(t) \cdot r_{v_{i}, \text { out }, i, j} \cdot \Phi_{v, \text { out }, i, j}\left(k_{i}(t), v_{i}(t), k_{j}(t), v_{j}(t)\right) \cdot v_{i}(t),
\end{aligned}
$$

where:

$$
\begin{aligned}
& k_{i}(t) \text { - traffic flow density in the i-th point, } \\
& v_{i}(t) \text { - traffic flow speed in the i-th point, } \\
& p_{i n, i, j}, p_{\text {out }, i, j} \text { - probabilities of flow splitting or connecting at some traffic line intersecting point } \\
& \mathrm{i}, \mathrm{j} \\
& r_{k_{i}, \text { in. } ., j, j}, r_{k_{i}, \text { out }, i, j}, r_{v_{i}, \text { in. } ., j, j}, r_{v_{i}, \text { out }, i, j} \text { - coefficients DMTF, }
\end{aligned}
$$


$\Phi_{k, i n, i, j}\left(k_{j}\left(t-\tau_{i, j}\right), v_{j}\left(t-\tau_{i, j}\right), k_{i}(t), v_{i}(t)\right), \Phi_{k, \text { out }, i, j}\left(k_{i}(t), v_{i}(t), k_{j}(t), v_{j}(t)\right)$ - nonlinear functions of DMTF,

$\Phi_{v, i n, i, j}\left(k_{j}\left(t-\tau_{i, j}\right), v_{j}\left(t-\tau_{i, j}\right), k_{i}(t), v_{i}(t)\right), \Phi_{v, \text { out }, i, j}\left(k_{i}(t), v_{i}(t), k_{j}(t), v_{j}(t)\right)$ - nonlinear functions of DMTF.

Nin, Nout - Line numbers that incoming or outgoing the $i^{\text {th }}$ point, respectively.

Vehicle quantity at each road element is:

$$
N_{\text {auto }, e}=\int_{x_{i}}^{x_{j}} k(x) d x,
$$

where:

$x_{i, j}$ - traffic line element boundary points,

$k_{i, j}$ - traffic flow density at boundary points.

The total number automobiles in the road are:

$$
N_{\text {total }}(t)=\sum_{e=1}^{N e} N_{\text {auto }, e},
$$

where: $N e$ - number of road elements.

\section{Numerical results of traffic flow dynamics}

Traffic flow movement in a road with one lane is analysed, when in the beginning and the end of a road, side conditions (Fig. 2) are known. Traffic light operates in one point of a road. Traffic flow parameter (traffic density and traffic flow speed) changes in time and every point of road are determined by using DMTF.

This method tries to show the effectiveness of the created DMTF, when modelling the complicated traffic flow dynamics.

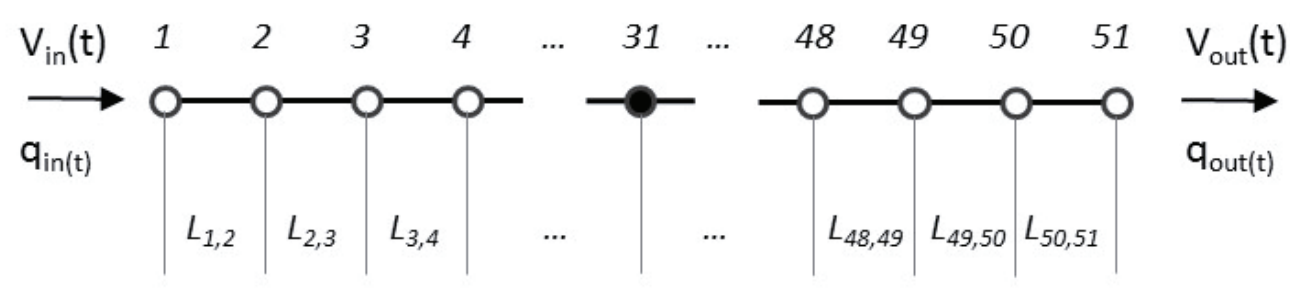

Fig. 2. Structure of one way road part creation

Node 31 is the node where a traffic light signal is entered as the function of time. The traffic light switching cycle is shown in the Fig. 3. The period of switching is equal 90 second.

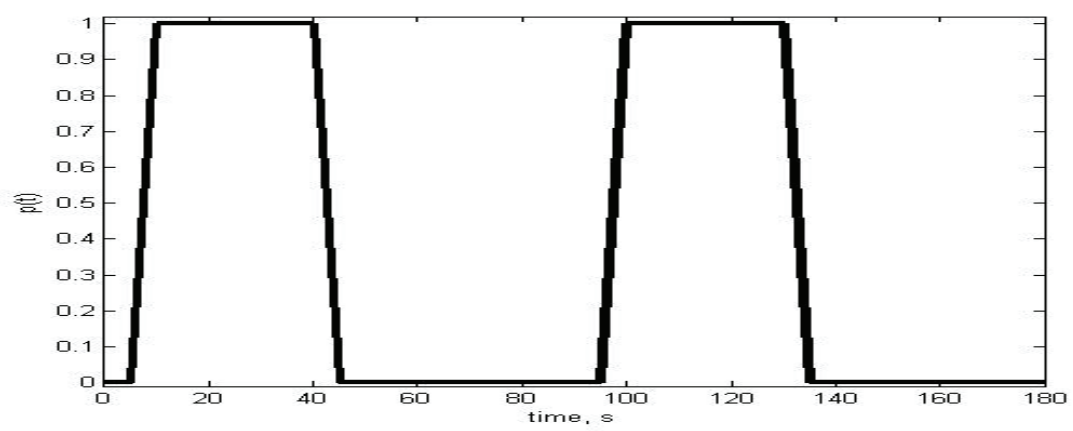

Fig. 3. The traffic light switching cycle 
The number of points is 51 (50 elements); length of road is $L=5000 \mathrm{~m}$ (Fig. 2). Boundary conditions at the first and final points are:

- Traffic flow rate: $q(x=0, t)=q_{\text {in }}=1.25$ auto $/ s q(x=L, t)=q_{\text {out }}=1.25$ auto $/ s$,

- Traffic flow velocity: $v(x=0, t)=v_{\text {in }}=12.5 \mathrm{~m} / \mathrm{s}=45 \mathrm{~km} / \mathrm{h}, v(x=L, t)=v_{\text {out }}=12.5 \mathrm{~m} / \mathrm{s}=45 \mathrm{~km} / \mathrm{h}$. Initial conditions of variables: $v_{i}(t=0)=12.5 \mathrm{~m} / \mathrm{s} ; k_{i}(t=0)=0.05$ auto $/ \mathrm{m} ; i=1, \ldots, 51$. Integration time step is $\Delta t=0.01 \mathrm{~s}$.

Changes of traffic flow parameters in time and road are shown in Fig. 4.
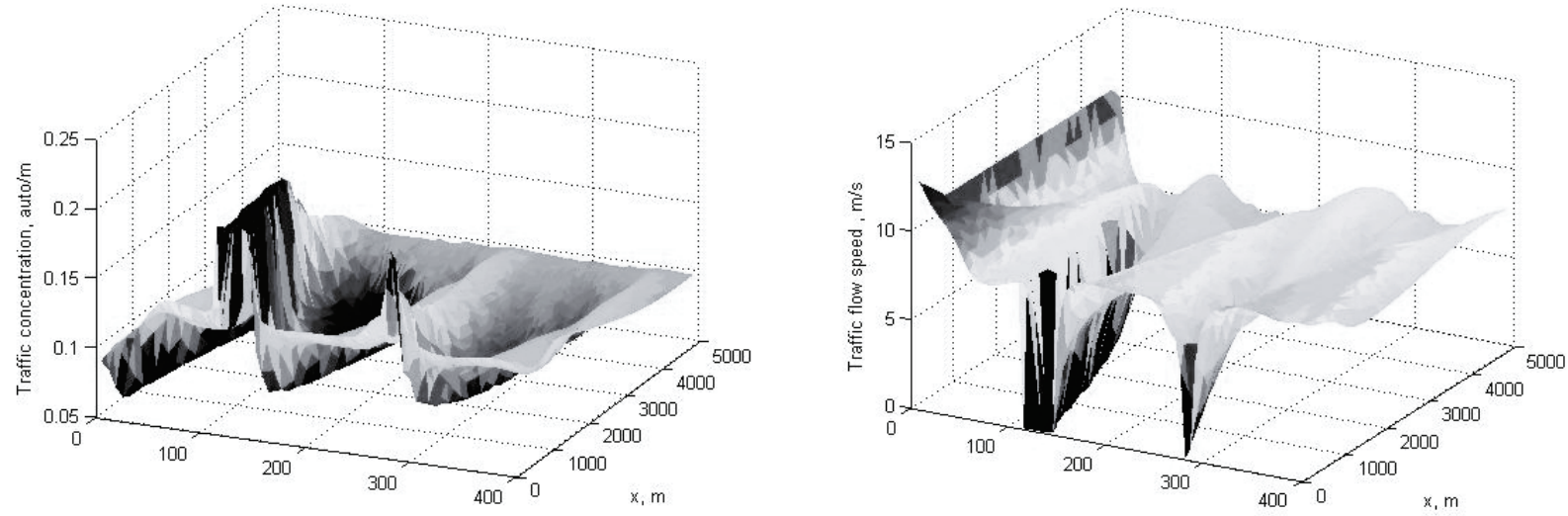

a)

$$
\text { time, s }
$$

b)

$$
\text { time, s }
$$

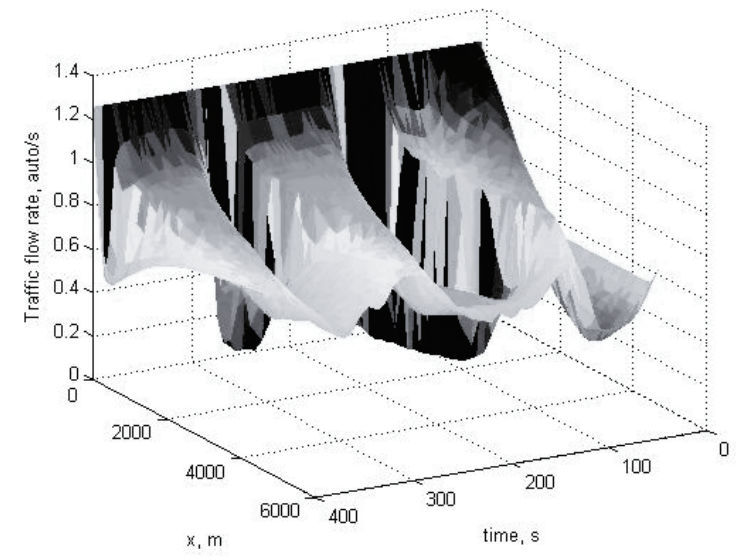

Fig. 4. Change of traffic flow density (a), speed (b) and traffic flow rate (c) upon time

The total number of automobiles in the road is shown in the Fig. 5.

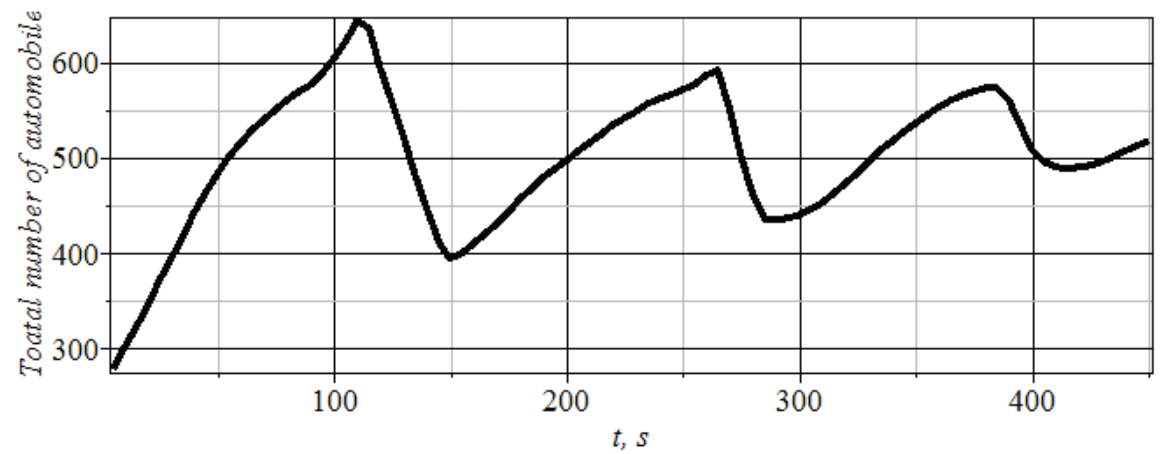

Fig. 5. Change of total number automobiles upon time 


\section{Conclusions}

The developed discrete model of traffic flow allows the consideration of traffic flow, as a dynamic system and to assess rapidly changing driving conditions. Using the created DMTF, traffic flow parameter (traffic flow density, traffic flow speed and traffic flow rate) changes in time and space are obtained. With the traffic-light working with a switching period of $90 \mathrm{~s}$, traffic flow density, traffic flow speed and traffic flow rate change at the same period and with a certain delay, which depends on road coordinates. By using this method, it is possible to model traffic flows with variable side and starting conditions, evaluating road geometry, allowed traffic flow density and traffic flow speed values. The mathematical experiment results are similar to experimental measuring data and behave normally in the points near traffic-lights.

\section{Acknowledgements}

This work has been supported by the European Social Fund within the project Development and application of innovative research methods and solutions for traffic structures, vehicles and their flows, project code VP1-3.1-ŠSMM-08-K-01-020.

\section{References}

[1] Chalons, C., Gotin, P., Godunov scheme and sampling technique for computing phase transitions in traffic flow modelling, Interface and Free Boundaries, pp. 197-221, 2008.

[2] Safanof, L. A., Tomer, E., Strygin, V.V., Havlin, S., Periodic solutions of a non-linear traffic model. Phyica A, pp.147-155, 2000.

[3] Kim, Y., Keller H., On-line traffic flow model applying the dynamic flow - density relation, Road transport information and control, P. 105, 2002.

[4] Liu, T., Jia, L., Zhu, W-x., A New traffic flow Model with the Effects of backward Looking and Relative Current, Fuzzy Systems and Knowledge Discovery, FSKD, Fifth International Conference on 5(DOI 10.1109/FSKD.2008.113), pp. 438-442, Jinan Shandong 2008.

[5] Flötteröd, G., Nagel K., High Speed Combined Micro/Macro Simulation of Traffic Flow, Proccedings of the 2007 IEEE Intelligent Transportation Systems conference, P. 6, 2007.

[6] Bonzani I., Hyperboliciy analyzis of a class of dynamical systems modelling traffic flow, Applied Mathematics Letters, Vol. 20(8), pp. 933-937, 2007.

[7] Nikolov, E., Traffic flow model based on the Green - function, Intelligent Systems, IS'08, $4^{\text {th }}$ International IEEE Conference 1 (DOI 10.1109/IS.2008.4670421), pp. 4-25-4-32, Varna 2008.

[8] Gning, A., Mihaylova, L., Boel, R., An interval compositional vehicular traffic model for Realtime applications, IEEE Inteligent Vehicle symposium, pp. 494-499, Eindhoven 2008.

[9] Li, L., Li-qun, X., Linear stability analysis of a multi-vehicle car-following traffic flow model, Management Science and Engineering, ICMSE 2008, 15th Annual Conference Proceedings, International Conference on (DOI 10.1109/ICMSE.2008.4669125), pp. 1642-1647, Long Beach, CA 2008

[10] Prigogine, I., Herman, R., Kinetic Theory of Vehicular Traffic, p. 101, New York 1971.

[11] Tampère, C. M. J., Human-kinetic multiclass traffic flow theory and modelling: with application to advanced driver assistance systems in congestion, Netherlands TRAIL Research School, p. 309, 2004.

[12] Helbing, D., Greiner A., Modelling and Simulation of Multi-Lane Traffic Flow, (arXiv:condmat/9806126), 1998.

[13] Karner, B. S., Klenov, S. L., Deterministic three-phase traffic flow models, Journal of Physics A: Mathematical And General, Vol. 39, pp. 1775-1809, 2006.

[14] Junevičius. R., Bogdevičius, M., Mathematical modelling of network traffic flow, Transport Vol. 24(4): 333-338, 2009. 